\title{
Too much of a good thing? An observational study of prolific authors
}

Elizabeth Wager, Sanjay Singhvi, Sabine Kleinert

Introduction: Researchers' productivity is usually measured in terms of their publication output. A minimum number of publications is required for some medical qualifications and professional appointments. However, authoring an unfeasibly large number of publications might indicate disregard of authorship criteria or even fraud. We therefore examined publication patterns of highly prolific authors in 4 medical specialties. Methods: We analysed Medline publications from 2008-12 using bespoke software to disambiguate individual authors focusing on 4 discrete topics (to further reduce the risk of combining publications from authors with the same name and affiliation). This enabled us to assess the number and type of publications per author per year. Results: While $99 \%$ of authors were listed on fewer than 20 publications in the 5-year period, 24 authors in the chosen areas were listed on at least 25 publications in a single year (i.e. $>1$ publication per 10 working days). Types of publication by the prolific authors varied but included substantial numbers of original research papers (not simply editorials or letters). Conclusions:

Institutions and funders should be alert to unfeasibly prolific authors when measuring and creating incentives for researcher productivity. 
$1 \quad$ Too much of a good thing? An observational study of prolific

2

\author{
authors
}

3 Elizabeth Wager

$4 \quad$ Sideview, Princes Risborough, UK

5

6 Sanjay Singhvi

$7 \quad$ SystemAnalytic, London, UK

8

9 Sabine Kleinert

10 The Lancet, London, UK

11

12

13

14 Corresponding author

15 Dr Elizabeth Wager, Sideview, 19 Station Road, Princes Risborough, HP27 9DE, UK

16 email: liz@sideview.demon.co.uk

17 phone: +44-1844-275814

18 fax: $+44-1844-275034$ 
Introduction

21 The productivity of researchers is usually measured in terms of the number of publications they

22 author. Some medical qualifications and academic appointments (e.g. 'habilitation' requirements

23 in several countries) require a minimum number of publications in recognised, peer-reviewed

24 journals (Buddeberg-Fischer, Stamm \& Buddeberg, 2009). Press releases announcing

25 institutional appointments often mention the appointee's publication record. An informal web

26 search produced examples mentioning that newly appointed individuals were already authors of

27300,400 or 1000 peer-reviewed publications [Elizabeth Wager, personal observation, data

28 presented at $7^{\text {th }}$ International Congress on Peer Review \& Biomedical Publication, Chicago,

29 2013]. One might therefore assume that the more publications a researcher is listed on, the better.

30 However, authoring an unfeasibly large number of publications might suggest guest authorship

31 or even fraud.

32

33 The link between extreme productivity and fraud is supported by anecdotal evidence. For

34 example, the physicist Jan Hendrik Schön produced about 40 research papers in one year

35 (submitting 7 in a single month), all of which were later retracted (Reich, 2009). Similarly, the

36 discredited anaesthetist Yoshitaka Fujii published 30 clinical trials in a single year (Tramèr,

37 2013). The phenomenon of senior researchers abusing their positions and demanding guest

38 authorship (i.e. listing despite making no, or minimal, contribution to the research) has also been

39 documented (Kwok, 2005; Shulkin et al, 1993).

40 
41 We therefore examined researchers' publication outputs to provide some initial insights into and 42 measurements of the phenomenon of prolific authorship.

\section{Methods}

49 Since simple searches of Medline by author name do not accurately identify individuals and cannot distinguish publications from different authors who have the same name, we used a

51 bespoke, semi-automated tool (developed by SystemAnalytic) that considers additional author

52 characteristics such as affiliation, publication history, and patterns of co-authorship. To further

53 reduce the chance of combining publications from different authors with the same name, we

54 focused on 4 discrete (arbitrarily chosen) topics (epilepsy, rheumatoid arthritis, renal

55 transplantion and liver transplantation) which were defined by keywords in the Medline

56 database. For each topic we analysed all publications listed on Medline from January 2008 to

57 December 2012. Using the software we characterized: the number of publications per individual,

58 the types of publication, and patterns of author order. We also manually checked outputs for a

59 convenience sample of the 10 most prolific authors for each topic using Medline to verify that

60 these did, indeed, appear to be from single authors and to check the types of publication and

61 authorship order.

62

63 Results 
64 We assessed 58,400 publications for 163,993 researchers. During the 5 years studied, $99 \%$ of 65 researchers $(162,744)$ were listed on fewer than 20 publications (Table 1). In contrast, the 66 median total number of publications (excluding letters and editorials) for the most prolific 67 authors was 93 (maximum 132, interquartile range 65-103). Considering individual years within 68 this period, the maximum number of publications per year was 43 for any type of publication and 6915 for clinical trials.

Detailed, manual inspection and analysis of the output of the 40 most prolific authors (i.e. the top 7210 for each topic) revealed great variations in the types of publications produced (Table 2). For example, one author published 32 letters and 22 review articles but only 1 primary research article reporting a clinical trial. Another published 34 reports of clinical trials and 19 reviews but only 3 letters. The authors' positions in the order of listing also varied, with several individuals featuring mainly as last author. The highest proportion of last author publications by an

77 individual was 93/105 (89\%), and the highest proportion of first author publications was 44/79

78 (56\%). Of the 40 most prolific authors, 24 were listed on at least 25 publications in any single 79 year (i.e. $>1$ publication per 10 working days).

81 The 40 most prolific authors were based in 5 continents (none from Africa) and a range of 82 countries, the most common being Germany (6), the Netherlands (6), and Japan (4). We did not 83 find any Medline retractions associated with these authors. 
86 Using specially designed software we were able to calculate the number of publications per

87 individual researcher and thus provide some measures of prolific authorship in a range of

88 medical fields. More detailed analysis also revealed the publication patterns of the most prolific

89 authors and we found these to be highly variable.

91 Judging by our manual analysis of the 40 most prolific authors, the software (originally designed 92 to analyse publication patterns of medical opinion leaders) successfully identified the output of 93 individual researchers. We limited our search to Medline and did not consider other publications 94 such as conference abstracts or those in non-listed journals. This means our estimates of prolific 95 output are conservative and may not be generalizable to other disciplines. This was an 96 exploratory, observational study to give an initial impression on the phenomenon of high author 97 productivity. We did not attempt to assess the causes for high productivity.

99 Since our initial study, another group has examined authorship patterns among diabetes

100 researchers and noted that the most prolific were named, on average, on 7 articles reporting

101 clinical trials per year, for 10 years (Holleman et al, 2015). One limitation both of our study and

102 that by Holleman et al is that neither attempted to determine the number of trials described in

103 these publications, so one explanation of some of the apparently prolific authors is that they were

104 involved mainly with very large studies that generated many publications (Wager, 2015).

105 However, the work required to take part in both the research and publication of such large trials

106 is still substantial and such high productivity raises questions, at the very least, about how

107 authorship guidelines are being interpreted. 
109 While special techniques are currently required to analyse the output of all biomedical

110 researchers, and thus get information about normal and abnormal productivity, it is

111 straightforward to assess the output of a small number of individuals and to validate this

112 manually. We therefore suggest that institutions and funders should be alert to the possibility of

113 excessive authorship. One simple technique would be to require job or research funding

114 applicants to include a total publication count in their application or CV. Spotting or verifying

115 over-prolific authors should become easier in future if journals and databases adopt researcher

116 identification systems such as ORCID (http://orcid.org) rather than relying simply on author

117 names for identification. Although the absolute number of highly prolific authors in each field is

118 probably small, asking researchers to justify their authorship, if there are any suspicions, shows

119 that institutions take research integrity seriously. Abusive authorship patterns, such as senior

120 figures who demand to be listed on publications despite having had little or no involvement in

121 research are well documented (Kwok, 2005) and can have damaging effects on junior researchers

122 because they send a signal that honest authorship is unimportant.

123

124 We suggest that institutional authorship policies and guidelines should stress the importance of

125 following accepted authorship criteria (which may differ between disciplines) and that

126 institutions should have systems in place to handle suspected abuses. Many guidelines note that

127 authorship entails accountability for the research being reported (ICMJE, 2013; Anon, 2007) and

128 this aspect should be reflected in policies and training. Institutions should also consider how to

129 reduce the 'publish or perish' atmosphere, often cited as a factor in misconduct and questionable

130 research practices, and how to create an environment that encourages integrity and honest 
131 authorship practices (Wager, 2015). Appointment and tenure committees should also develop

132 methods to measure the quality rather than merely the quantity of a researcher's publications.

133 


\section{Author contributions / Acknowledgments}

136 EW had the idea for the study, designed it jointly with the other authors, did the manual

137 assessments of the most prolific authors and the descriptive statistics, drafted the article, had full

138 access to all the data and takes responsibility for the integrity of the data and the accuracy of the

139 data analysis. SS contributed to the design and reporting of the study and provided the

140 bibliometric analyses. SK contributed to the study design, interpretation, and reporting.

141

142

143 Data access statement

144 Anonymised data are available from the corresponding author, on request. 


\section{References}

148 Anon. 2007. Who is accountable? Nature 450:1

149 Buddeberg-Fischer B, Stamm M, Buddeberg M.2009. Academic career in medicine -

150 requirements and conditions for successful advancement in Switzerland. BMC Health

$151 \quad$ Services Research 9:70

152 Holleman F, Uijldert M, Donswijk LF, Gale EAM. 2015. Productivity of authors in the field of

153 diabetes: bibliographic analysis of trial publications. BMJ 351:h2638.

154 International Committee of Medical Journal Editors. 2013. Recommendations for the conduct, 155 reporting, editing, and publication of scholarly work in medical journals.

$156 \mathrm{http} / / / \mathrm{www} . \mathrm{icmje}$. org/recommendations/browse/roles-and-responsibilities/defining-the-

157 role-of-authors-and-contributors.html\#two (accessed 23 May 2015)

158 Kwok LS. 2005. The White Bull effect: abusive coauthorship and publication parasitism. J Med $159 \quad$ Ethics 31:554-556

160 Reich ES. 2009. The rise and fall of a physics fraudster. Physics World, May 2009:24-29

$161 \quad$ http://www.nasw.org/users/essreich/SchonArticlePhysicsWorld.pdf

162 Shulkin DJ, Goin JE, Rennie D. 1993. Patterns of authorship among chairmen of departments of 163 medicine. Acad Med 68:688-692

164 Tramèr M. The Fujii story. A chronicle of naïve disbelief. 2013. Eur J Anaesthesiol 30:195-8

165 Wager E. 2015. Are prolific authors too much of a good thing? BMJ 351:h2782

166

167

168 


\section{Table $\mathbf{1}$ (on next page)}

Productivity of researchers in four selected topics indicated by the number of Medline publications 2008-12 per author and the maximum number for any one individual (Max) 
3 Table 1

4 Productivity of researchers in four selected topics indicated by the number of Medline

5 publications 2008-12 per author and the maximum number for any one individual (Max)

6

\begin{tabular}{lccccc}
\hline Topic & \multicolumn{7}{c}{ Number of publications/author 2008-12 $(\mathrm{N}, \%)$} \\
\hline $1-20$ & $21-30$ & $31-40$ & $41-50$ & $>50$ & Max
\end{tabular}

\begin{tabular}{lcccccc}
\hline Epilepsy & 63,866 & 141 & 34 & 11 & 37 & 118 \\
& $(99.7)$ & $(0.2)$ & $(0.05)$ & $(0.02)$ & $(0.06)$ & \\
Rheumatoid & 33,953 & 124 & 66 & 30 & 41 & 149 \\
arthritis & $(98.8)$ & $(0.4)$ & $(0.2)$ & $(0.08)$ & $(0.1)$ & 123 \\
Renal & 38,575 & 201 & 62 & 34 & 38 & 128 \\
transplant & $(99.1)$ & $(0.5)$ & $(0.2)$ & $(0.1)$ & $(0.1)$ & \\
Liver & 26,350 & 174 & 69 & 36 & 56 & $(0.2)$ \\
transplant & $(98.7)$ & $(0.7)$ & $(0.3)$ & $(0.1)$ & & \\
\hline
\end{tabular}


Table 2 (on next page)

Types of publication and author order for the 10 most prolific authors in 4 medical specialties over 5 years (2008-12) on Medline

Auth ID = author identifier (rank of top 10 most prolific authors) Clin trial $=$ report of a randomized clinical trial Res art $=$ research article Case rep $=$ case report Syst rev $=$ systematic review 


\section{Table 2}

3 Types of publication and author order for the 10 most prolific authors in 4 medical specialties over 5

4 years (2008-12) on Medline

\begin{tabular}{|c|c|c|c|c|c|c|c|c|c|c|c|c|}
\hline \multicolumn{4}{|c|}{ Publication types } & & & & & & \multicolumn{4}{|c|}{ Author position $(\mathrm{N}(\%)$} \\
\hline $\begin{array}{l}\text { Auth. } \\
\text { ID }\end{array}$ & $\begin{array}{l}\text { Clin } \\
\text { trial }\end{array}$ & Res art. & $\begin{array}{l}\text { Case } \\
\text { rep. }\end{array}$ & Editorial & Letter & $\begin{array}{c}\text { Syst. } \\
\text { rev. }\end{array}$ & Review & Total & $\begin{array}{l}\text { Max/ } \\
\text { year }\end{array}$ & $1 \mathrm{st}$ & Middle & Last \\
\hline 1 & 61 & 37 & 7 & 7 & 11 & 6 & 20 & 149 & 38 & $16(11)$ & $79(53)$ & $54(36)$ \\
\hline 2 & 26 & 62 & 4 & 1 & 13 & 7 & 25 & 138 & 43 & $1(1)$ & $106(77)$ & $31(22)$ \\
\hline 3 & 34 & 72 & 4 & 3 & 3 & 3 & 19 & 138 & 35 & $6(4)$ & $77(56)$ & $55(40)$ \\
\hline 4 & 6 & 83 & 25 & 0 & 8 & 0 & 6 & 128 & 31 & $3(2)$ & $47(37)$ & $78(61)$ \\
\hline 5 & 46 & 45 & 5 & 2 & 18 & 1 & 7 & 124 & 27 & $0(0)$ & $103(83)$ & $21(17)$ \\
\hline 6 & 10 & 66 & 19 & 4 & 13 & 0 & 8 & 120 & 31 & $17(14)$ & $101(84)$ & $2(2)$ \\
\hline 7 & 26 & 62 & 12 & 2 & 7 & 0 & 10 & 119 & 25 & $1(1)$ & $64(54)$ & $54(45)$ \\
\hline 8 & 1 & 56 & 3 & 4 & 32 & 0 & 22 & 118 & 30 & $0(0)$ & $70(59)$ & $48(41)$ \\
\hline 9 & 7 & 82 & 9 & 1 & 3 & 2 & 9 & 113 & 33 & $5(4)$ & $79(70)$ & $29(26)$ \\
\hline 10 & 41 & 20 & 29 & 0 & 10 & 1 & 11 & 112 & 27 & $7(6)$ & $46(41)$ & $59(53)$ \\
\hline
\end{tabular}

5

6 Auth ID = author identifier (rank of top 10 most prolific authors)

$7 \quad$ Clin trial $=$ report of a randomized clinical trial

$8 \quad$ Res art $=$ research article

9 Case rep $=$ case report

10 Syst rev $=$ systematic review 